\title{
PROGRAMA PIONERO DE SIMULACIÓN EN SUTURA PARA ESTUDIANTES DE MEDICINA DE PREGRADO*
}

\author{
Dr. Juan Alvarado I. ${ }^{1}$, Int. Juan Pablo Henríquez R. ${ }^{2}$, \\ Dr. Richard Castillo R. ${ }^{1}$, Al. Javiera Sosa B. ${ }^{2}$, Drs. Felipe León F. ${ }^{1}$, \\ Julián Varas C. ${ }^{1}$, Mauricio Camus A. ${ }^{3}$, Arnoldo Riquelme P. ${ }^{4}$, \\ Fernando Crovari E. ${ }^{1}$, Jorge Martínez C. ${ }^{1}$, Camilo Boza W. ${ }^{1}$, Nicolás Jarufe C. ${ }^{1}$
}

\author{
Departamento de Cirugía Digestiva, División de Cirugía. \\ Escuela de Medicina. \\ Departamento de Cirugía Oncológica, División de Cirugía. \\ ${ }^{4}$ Departamento de Gastroenterología y Centro de Educación Médica. \\ Pontificia Universidad Católica de Chile. \\ Santiago, Chile.
}

\section{Abstract \\ A pioneer simulation curriculum of suture technique training for medical students}

Introduction: Simulation has demonstrated utility in suture skills training on surgical residents. Objective: Evaluate validity of a simulated training curriculum of basic suture skills for medical students. Methods: Study conducted on 2013. Medical students were selected from the School of Medicine of Pontificia Universidad Católica de Chile (EMPUC). The program included four theoretical sessions and six practical sessions in order to training basic surgical tasks (five in total) on a simulated rubber model: one handed knot-tying, simple suture, vertical mattress suture, subcuticular interrupted suture and subcuticular running suture. A survey was applied to the participants to qualify each element of the program, on a scale of 1 to 7 (face-validity). One task (simple suture) was recorded on video, before and after training, to be evaluated by two experts on a blind fashion (content validity). Experts measure participant skills using an OSATS (Objective Structured Assessment of Technical Skills) and operative time. Statistical analysis for non-parametric variables, median and interquartile range, significant $p$-value $<0.05$. Results: Thirty-six medical students participated, with assistance percentage was over $80 \%$. Students qualified the experience favorably: simulated bench-model 6.0 [5.0-6.0], assistant-students 6.5 [6.0-7.0], practical sessions 7.0 [6.0-7.0], and general evaluation 6.5 [6.0-7.0]. In the evaluation of simple suture, there was a significant improvement on OSATS (10 [5-17] vs 19 [17-21]; $\mathrm{p}<0.001)$ and decrease of operative time (138 seg [101-185] vs 77 seg [67-89]; $p<0.001)$ after training in every participant. Conclusion: This simulated curriculum delivers an alternative method for the development of technical competencies in medical students and could be incorporated on medical schools curricula.

Key words: Education, medical, simulation, teaching, surgery, training/courses.

*Recibido el 24 de noviembre de 2014 y aceptado para publicación el 5 de marzo de 2015.

Este estudio fue financiado por proyecto FONDECYT Número 1141196 (N.J.) y 1120652 (A.R.)

Los autores no tienen conflicto de interés que reportar.

Correspondencia: Dr. Nicolás Jarufe C. njarufe@med.puc.cl 


\section{Resumen}

Introducción: La simulación ha demostrado ser útil en la enseñanza de técnicas de sutura en cirujanos en formación. Objetivo: Evaluar la validez de un programa de entrenamiento simulado de técnica básica de sutura para estudiantes de pregrado de medicina. Material y Métodos: Estudio experimental realizado el año 2013, se seleccionó estudiantes de pregrado de la Escuela de Medicina de la Pontificia Universidad Católica de Chile (EMPUC). El programa incluye 4 sesiones teóricas y 6 prácticas, entrenando 5 tareas en modelo simulado de goma: nudo manual y puntos simple, Donati, intradérmico interrumpido e intradérmico continuo. Se entregó una encuesta a los participantes para calificar cada elemento del curso, en escala de 1 a 7 (validez de apariencia). Se grabó en vídeo una tarea (punto simple), pre y post entrenamiento, para ser evaluadas por 2 cirujanos en forma ciega (validez de contenido). Se utilizó escalas OSATS y medición de tiempo operatorio. Análisis estadístico para variables no paramétricas, mediana y rango interquartil, valor significativo $\mathrm{p}<0,05$. Resultados: Participaron 36 estudiantes, con un porcentaje de asistencia mayor a un $80 \%$. Los alumnos calificaron favorablemente la experiencia: simulador de mesa 6,0 [5,0-6,0], ayudantesalumnos 6,5 [6,0-7,0], clases prácticas 7,0 [6,0-7,0] y evaluación general 6,5 [6,0-7,0]. Respecto al punto simple, hubo mejoría significativa en OSATS $(10$ [5-17] vs 19 [17-21]; $\mathrm{p}<0,001)$ y disminución de tiempo operatorio (138 seg [101-185] vs $77 \mathrm{seg}$ [67-89]; $\mathrm{p}<0,001)$ al finalizar el entrenamiento. Conclusión: Este programa simulado plantea un método alternativo para el desarrollo de competencias técnicas y podría ser incorporado en los currículos formales de las escuelas de medicina.

Palabras clave: Educación médica, simulación, enseñanza, cirugía, cursos de entrenamiento.

\section{Introducción}

Las técnicas de sutura son esenciales para el ejercicio de la profesión de médico general ${ }^{1}$, particularmente en la atención de urgencias médicoquirúrgicas. Son parte de las competencias mínimas de un médico recién egresado descritas, tanto a nivel nacional como internacional (The Tuning Proyect ${ }^{2,3}$, programa MECESUP ${ }^{4}$.

Estas destrezas técnicas son tradicionalmente enseñadas a través del modelo tutorial, que consiste en realizar un procedimiento clínico bajo supervisión docente, por lo que no siempre es posible que su enseñanza sea estandarizada. El modelo tutorial se ve limitado, actualmente, por diversas situaciones como la seguridad del paciente, disponibilidad docente, espacio físico, exposición a patología de urgencia, evaluaciones subjetivas, entre otros ${ }^{5}$. Las escuelas de medicina del país, por lo tanto, se ven con diversas dificultades a la hora de asegurar que estas competencias mínimas sean adquiridas por cada uno de sus alumnos. Se necesitan otras vías de aprendizaje y evaluación para asegurar la adquisición de estas habilidades sin exponer a los pacientes a un riesgo innecesario.

La simulación ha permitido la adquisición de competencias técnicas en cirugía tradicional abierta $^{6-8}$ y laparoscópica para postgrado ${ }^{9-12}$. Para su demostración se utilizan pautas que miden objetivamente la adquisión de habilidades, evaluando la técnica quirúrgica aprendida bajo una escala de puntaje, como el OSATS (Objective Structured Assessment of Technical Skills) ${ }^{6}$. El uso de estas pautas, junto a metodología docente especializada en simulación, han permitido desarrollar programas de entrenamiento fiables $\mathrm{y}$ válidos ${ }^{13}$, en términos de apariencia, contenido, constructo y validez predictiva, con resultados reproducibles (validez externa). Los programas de entrenamiento simulado permiten optimizar recursos, especialmente docente y horario, aumentar la práctica técnica, y acortar curvas de aprendizaje, constituyendo un modo seguro, estandarizado y validado de aprendizaje para todos los entrenados ${ }^{5}$.

Los niveles iniciales de un modelo experimental corresponden a la validez de apariencia y de contenido, donde se evalúa el grado en que la situación simulada se asemeja al mundo real y si el contenido del ejercicio simulado puede ser medido. La validez de constructo y predictiva, por su parte, corresponden a los niveles de validez más altos y difíciles de alcanzar en un modelo experimenta ${ }^{14} \mathrm{y}$ hacen referencia a si la situación simulada permite discriminar entre distintos participantes, por ejemplo, novatos de expertos, y si es que la medición puede extrapolarse a la situación real.

En vista del éxito del uso de simulación en programas de postgrado de la Escuela de Medicina de la Pontificia Universidad Católica de Chile (EMPUC), se plantea la posibilidad de enseñar técnicas de sutura básica a estudiantes de pregrado en un modelo simulado. La Academia de Cirugía UC (asociación de estudiantes de pregrado de la EMPUC enfocada al desarrollo científico de temas quirúrgicos), en colaboración con docentes de la misma facultad, desarrollaron un programa pionero de entrenamiento 
simulado de sutura con la intención de adquirir destrezas por medio de esta herramienta educacional.

El objetivo de este estudio es evaluar la validez de apariencia y de contenido en un programa pionero de entrenamiento simulado de técnica básica de sutura.

\section{Materiales y Métodos}

\section{Participantes}

Se seleccionaron estudiantes de tercero a quinto año de la Escuela de Medicina de la Pontificia Universidad Católica de Chile, con escasa o nula práctica clínica en sutura. Los alumnos se inscribieron de manera voluntaria a participar del curso en horario extracurricular.

\section{Metodología de trabajo}

Se desarrolló el programa de entrenamiento entre julio y diciembre de 2013, compuesto de seis sesiones prácticas presenciales, y cuatro sesiones teóricas.

Se realizó una sesión práctica al mes, cada una de 100 min de duración. En cada sesión se entrenaron 5 tareas $^{7}$ : punto simple interrumpido, punto Donati o colchonero vertical, punto invertido (intradérmico interrumpido con nudo en profundidad), sutura intradérmica (intradérmico continuo) y anudado con mano. Estas sesiones fueron dirigidas por 2 cirujanos residentes de la EMPUC y 8 ayudantes-alumnos previamente entrenados en estas tareas. Este cuerpo docente entregó retroalimentación efectiva ${ }^{15,16}$ a cada participante, con el objeto de corregir sus errores inmediatamente y optimizar su entrenamiento. El protocolo se construyó en base al modelo de debriefing plus-delta, considerando el ciclo de aprendizaje de Kolb, que incorpora el análisis de las experiencias en el aprendizaje activo del adulto ${ }^{17,18}$.

En las sesiones teóricas se reforzó el contenido necesario para ser aplicado en las sesiones prácticas. Este material se publicó en una página web ${ }^{19}$ diseñada para facilitar el estudio y se abarcaron los siguientes temas: "Materiales y técnicas en sutura clásica", "Características de heridas y manejo inicial", "Manejo quirúrgico básico de heridas", "Temas de cirugía menor". Se solicitó a cada participante el estudio formal de cada clase que luego se evaluó en una prueba.

Como requisito de aprobación se consideró una asistencia mínima de $80 \%$ a las clases prácticas y obtener una calificación mínima de $60 \%$ en la prueba de conocimientos teóricos.

\section{Materiales}

Se utilizó un simulador de mesa, basado en un polímero sintético de alta densidad (conocida comercialmente como "goma esponja") (Figura 1). Para ello se montaron 2 trozos de goma de $5 \times 15 \mathrm{~cm}$ y 10 $\mathrm{x} 15 \mathrm{~cm}$, de $2 \mathrm{~cm}$ de espesor, con incisiones longitudinales de $10 \mathrm{~cm}$. El polímero se fijó sobre una tabla de madera de $25 \times 25 \mathrm{~cm}$. Para los puntos se utilizó un porta aguja, una pinza anatómica y 12 suturas de Seda 2-0 (Ethicon ${ }^{\circledR}$, Johnson \& Johnson) por cada estudiante. Para el anudado se utilizó monofilamento (hilo de nylon).

\section{Costos}

El costo de materiales por alumno para realizar el curso completo fue de $\$ 16.650$ clp aproximadamente, determinado principalmente por el modelo de caja, instrumental y material de sutura. Esta estimación no considera la infraestructura ni horas docentes requeridas.

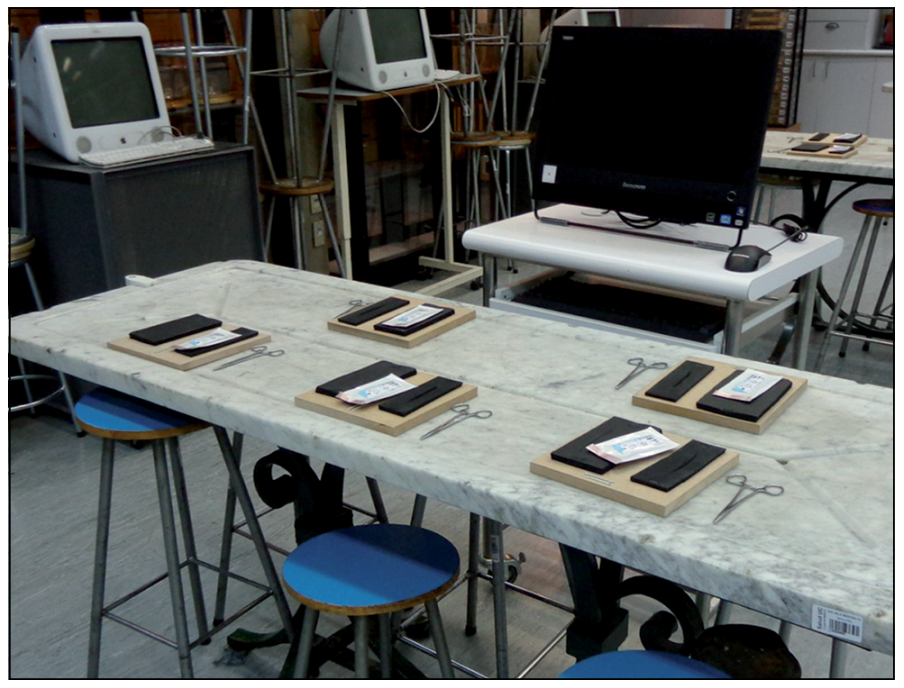

Figura 1. Modelo simulado de mesa para el entrenamiento de técnica básica en sutura. 


\section{Evaluaciones}

Para evaluar la validez de apariencia, se aplicó una encuesta a los participantes que consideraba elementos subjetivos del curso, entre los cuales se encuentran: simulador de mesa, desempeño de ayudantes-alumnos, clases prácticas y evaluación general del curso. Cada elemento fue valorado en una escala de 1 a 7: una calificación de "1" refleja completa insatisfacción, mientras que "7" significa que el elemento evaluado es excelente y muy aceptado. Además se preguntó por la importancia del rol de los ayudantes-alumnos y el grado de satisfacción por los logros obtenidos. La encuesta se aplicó al finalizar la primera clase práctica y al término del curso para determinar cambios en la apreciación.

Para evaluar validez de contenido, cada alumno fue grabado realizando la tarea de punto simple, en el modelo descrito, antes y después del programa de entrenamiento. Las demás tareas sólo fueron entrenadas y no evaluadas. Dos cirujanos ciegos al alumno y al momento de grabación, analizaron estos vídeos y aplicaron pautas OSATS generales, previamente validadas ${ }^{6,10}$. Las pautas OSATS generales constan de los siguientes elementos, evaluados en una escala Likert de 1 a 5: manejo de instrumental, respeto por el tejido, tiempo de movimientos y fluidez, flujo de operación y planificación y conocimientos específicos. Además se registró el tiempo de cada procedimiento, medido en segundos.

El protocolo incluyó en su diseño el concepto de evaluación programática que considera el impacto de la evaluación en el aprendizaje activo de los alumnos, cambiando el paradigma de "evaluación de lo aprendido" por "evaluación para el aprendizaje" 20 .

\section{Análisis estadístico}

Los datos fueron analizados utilizando el programa Statistical Package for the Social Sciences (SPSS) versión $20 \AA$. Las variables evaluadas correspondieron a OSATS y tiempo, mostradas como mediana y rango intercuartil (p25-p75). Se analizaron los datos pre-post con test de Wilcoxon para variables pareadas no paramétricas y test de Mann-Whitney para variables independientes no paramétricas. Se consideró estadísticamente significativo a un valor $p$ menor a 0,05 .

\section{Resultados}

Se conformó un grupo de 36 participantes quienes realizaron el programa de entrenamiento en su totalidad. Todos ellos contaron con un porcentaje de asistencia mayor al $80 \%$ en las clases prácticas y todos aprobaron la evaluación teórica.

\section{Validez de apariencia}

Se obtuvo la respuesta de 34 encuestas en primera instancia y 32 al finalizar el curso (Tabla 1). Las clases prácticas obtuvieron la más alta puntuación, mientras que el simulador de mesa obtuvo la menor calificación. El 97\% de los participantes refiere que los ayudantes-alumnos fueron fundamentales para el desarrollo del curso. Todos los alumnos están satisfechos por sus logros obtenidos.

\section{Evaluación de punto simple}

Los participantes, al realizar un punto simple, obtuvieron una mediana de puntaje OSATS pre entrenamiento de 10 [5-17] y post entrenamiento de 19 [17-21], con un $p<0,001$ (Figura 2). La mediana del tiempo de procedimiento pre entrenamiento fue de 138 segundos [101-185], mientras que en el post entrenamiento fue de 77 segundos [67-89], $\mathrm{p}<0,001$ (Figura 3).

Tabla 1. Resultados de la encuesta de apreciación del curso aplicada a los participantes. Se evaluó cada elemento en escala de 1 a 7 . Resultados presentados como mediana y rango intercuartil [p25-p75]

\begin{tabular}{|lcccc|}
\hline & $\begin{array}{c}\text { Evaluación al } \\
\text { inicio } \\
\mathbf{n}=\mathbf{3 4}\end{array}$ & $\begin{array}{c}\text { Evaluación del } \\
\text { Curso al final } \\
\mathbf{n}=\mathbf{3 2}\end{array}$ \\
\hline Clases prácticas & & - & 7,0 & {$[6,0-7,0]$} \\
\hline Simulador de mesa & 6,0 & {$[5,0-6,0]$} & 6,0 & {$[5,0-6,0]$} \\
\hline Ayudantes-alumnos & 7,0 & {$[6,0-7,0]$} & 6,5 & {$[6,0-7,0]$} \\
\hline Evaluación general & 6,5 & {$[6,0-7,0]$} & 6,5 & {$[6,0-7,0]$} \\
\hline
\end{tabular}

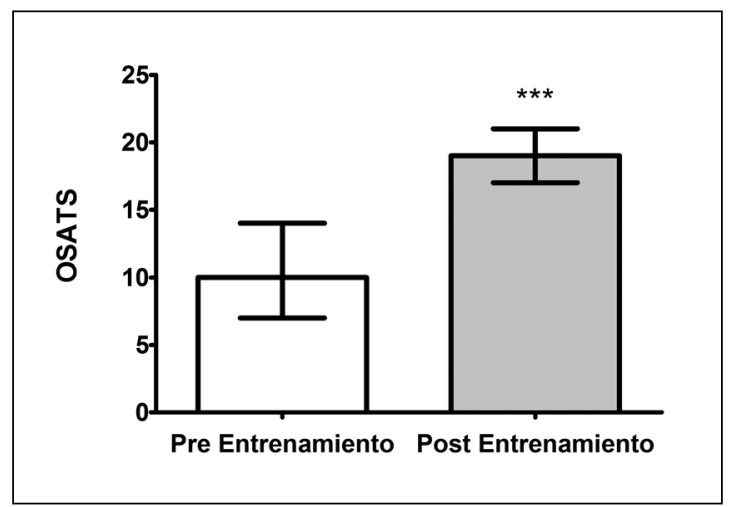

Figura 2. Comparación de puntajes OSATS (Objective Structured Assessment of Technical Skills) pre y post entrenamiento (punto simple). Se observa una mejoría significativa de puntaje OSATS en el post entrenamiento $(\mathrm{p}<0,001)$. 


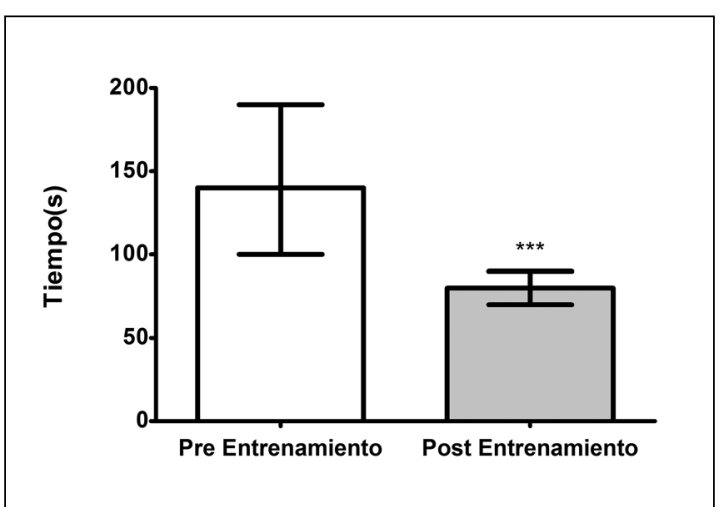

Figura 3. Comparación del tiempo de procedimiento (punto simple) pre y post entrenamiento. Se observa una disminución significativa de tiempo en el post entrenamiento $(\mathrm{p}<0,001)$. (s): segundos.

\section{Discusión}

El desarrollo de este programa demostró que es posible entrenar técnicas de sutura básica en simulación, de manera generalizada, estandarizada y en un tiempo de entrenamiento definido. Se utilizó materiales de bajo costo y tuvo un alto grado de satisfacción por parte de los participantes. Todos estos elementos permiten reproducibilidad y factibilidad.

Todos los participantes lograron cambios de habilidad en un tiempo total de $10 \mathrm{~h}$ de entrenamiento (distribuido en 6 meses), los rendimientos alcanzados por todos ellos son significativos, evidenciando la adquisición estandarizada de estas competencias. Para lograr estos resultados, fue necesario la aplicación de metodología docente como un programa estructurado con objetivos claros y uso de feedback efectivo ${ }^{16}$. Estos factores han sido propuestos como elementales en el desarrollo de programas de aprendizaje basado en competencias ${ }^{21}$.

En este curso se observó un alto porcentaje de asistencia a las clases teóricas, pese al horario extracurricular; además, las calificaciones para cada uno de los elementos evaluados del curso por parte de los participantes son cercanas al máximo y consistentes a través del tiempo. Todo esto, refleja el alto grado de satisfacción por parte de los alumnos respecto a los resultados obtenidos y a la forma de entrenamiento (aprendizaje). Este programa demuestra que es posible entrenar un volumen no menor de participantes (36 alumnos en total).

Las pautas OSATS utilizadas han sido validadas ampliamente en la literatura internacional ${ }^{22}$, principalmente en profesionales titulados con algún grado de formación en habilidades quirúrgicas (residentes, médicos, cirujanos). Su aplicación en pregrado, con alumnos que no cuentan con un basal de destrezas quirúrgicas, no está ampliamente difundido $\mathrm{y}$, en este estudio, se demostró que su aplicación en este contexto también permite discriminar grados de habilidades (validez de contenido). El uso de pautas OSATS facilita la estandarización de las destrezas adquiridas, pero requiere de tutores familiarizados con ellas.

Se observó que es posible generar un programa de entrenamiento con gastos relativamente bajos por concepto de insumos, basado en materiales simples y un modelo de caja de bajo costo. Además se optimizó el número de docentes requeridos, 1 tutor por cada 5 alumnos. Los ayudantes-alumnos entrenados lograron ser útiles en esta experiencia, con un alto grado de aceptación por parte de los participantes.

Este estudio no está exento de limitaciones. No se logró recopilar todas las encuestas de apreciación debido a dificultades con el seguimiento, considerando que el curso se realizó en horario extracurricular. Pudo haberse logrado un mayor grado de validez, ya que en esta experiencia no se logró diferenciar a expertos de novatos en el modelo de caja propuesto; validez de constructo $^{22}$. Además, dado que no se evaluó las demás tareas incluidas en el entrenamiento por dificultades técnicas, sería interesante evaluar en futuros estudios los cambios de destrezas en éstas, teniendo en cuenta que el punto simple es la tarea más representativa en términos de sutura.

Dado que los resultados del curso son dependientes de un número importante de docentes, se ha propuesto el uso de vídeos tutoriales autoinstructivos de tal manera de optimizar los recursos manteniendo su efectividad ${ }^{23}$, con lo que tendría un mayor atractivo para las escuelas de medicina del país. Por último, queda pendiente evaluar la transferencia de estas habilidades a situaciones clínicas con pacientes (validez predictiva) ${ }^{13}$, aunque existe en la literatura reportes que muestran que es posible que estas destrezas se transfieran a modelos más complejos ${ }^{6,24}$.

En conclusión, este programa de entrenamiento simulado de técnicas básicas de sutura es factible de realizar, siendo reproducible y válido en términos de apariencia y contenido. Todos los alumnos logran destrezas, demostrado a través de la mejoría significativa del puntaje OSATS y disminución del tiempo de procedimiento. Es necesario continuar esta línea de investigación para generar un programa de mejor diseño y validez (lograr validez de constructo y predictiva), posible de implementar en las diversas mallas curriculares de las escuelas de pregrado de medicina del país. 


\section{Referencias}

1. Proyecto MECESUP PUC 410. Desarrollo y evaluación de un perfil común del egresado de las escuelas de medicina de Chile. [Internet]. [cited 2014 Jul 15]. Available from: http://analisis.ufro.cl/index.php?option=com_con tent $\&$ view $=$ article $\&$ id $=144$ :puc0410\&catid $=45$

2. Cumming A, Cumming A, Ross M. The Tuning Project for Medicine-learning outcomes for undergraduate medical education in Europe. Med Teach. 2007;29:63641.

3. Tuning Project (Medicine). Learning outcomes/competences for undergraduate medical education in Europe [Internet]. [cited 2014 Oct 12]. Available from: http:// www.tuning-medicine.com/index.asp.

4. Preview Propuesta de Perfil del Egresado ASOFAMECH [Internet]. [cited 2014 Oct 12]. Available from: https://www.u-cursos.cl/commed/2007/0/INNCURRME/9/material_docente/previsualizar?id_material $=138916$.

5. Reznick RK, MacRae H. Teaching surgical skillschanges in the wind. N Engl J Med. 2006;355:2664-9.

6. Martin JA, Regehr G, Reznick R, MacRae H, Murnaghan $\mathrm{J}$, Hutchison $\mathrm{C}$, et al. Objective structured assessment of technical skill (OSATS) for surgical residents. Br J Surg. 1997;84:273-8.

7. Scott DJ, Goova MT, Tesfay ST. A cost-effective proficiency-based knot-tying and suturing curriculum for residency programs. J Surg Res. 2007;141:7-15.

8. Goova MT, Hollett LA, Tesfay ST, Gala RB, Puzziferri $\mathrm{N}$, Kehdy FJ, et al. Implementation, construct validity, and benefit of a proficiency-based knot-tying and suturing curriculum. J Surg Educ. 2008;65:309-15.

9. Hyltander A, Liljegren E, Rhodin PH, Lönroth $\mathrm{H}$. The transfer of basic skills learned in a laparoscopic simulator to the operating room. Surg Endosc Interv Tech. 2002;16:1324-8.

10. Aggarwal R, Boza C, Hance J, Leong J, Lacy A, Darzi A. Skills acquisition for laparoscopic gastric bypass in the training laboratory: an innovative approach. Obes Surg. 2007;17:19-27.

11. Corvetto M, Bravo MP, Montaña R, Utili F, Escudero E, Boza C, y cols. Simulación en educación médica: una sinopsis. Rev Med Chile 2013;141:70-9.

12. Fonseca AL, Evans LV, Gusberg RJ. Open surgical simulation in residency training: a review of its status and a case for its incorporation. J Surg Educ. 2013;70:12937.

13. Van Nortwick SS, Lendvay TS, Jensen AR, Wright
AS, Horvath KD, Kim S. Methodologies for establishing validity in surgical simulation studies. Surgery 2010;147:622-30.

14. Kane MT. Validating the Interpretations and Uses of Test Scores. J Educ Meas. 2013;50:1-73.

15. Varas J, Mejía R, Riquelme A, Maluenda F, Buckel E, Salinas J, et al. Significant transfer of surgical skills obtained with an advanced laparoscopic training program to a laparoscopic jejunojejunostomy in a live porcine model: feasibility of learning advanced laparoscopy in a general surgery residency. Surg Endosc. 2012;26:348694.

16. Norcini J, Burch V. Workplace-based assessment as an educational tool: AMEE Guide No. 31. Med Teach. 2007;29:855-71.

17. Kosir MA, Fuller L, Tyburski J, Berant L, Yu M. The Kolb learning cycle in American Board of Surgery InTraining Exam remediation: the Accelerated Clinical Education in Surgery course. Am J Surg. 2008;196:65762.

18. Levett-Jones T, Lapkin S. A systematic review of the effectiveness of simulation debriefing in health professional education. Nurse Educ Today 2014 Jun;34(6):e58-63. doi:10.1016/j.nedt.2013.09.020. Epub 2013 Oct 15

19. Academia de Cirugía UC [Internet]. [cited 2014 Nov 5]. Available from: http://acuc.cl/

20. Van der Vleuten CPM, Schuwirth LWT, Driessen EW, Dijkstra J, Tigelaar D, Baartman LKJ, et al. A model for programmatic assessment fit for purpose. Med Teach 2012;34:205-14

21. Kolozsvari NO, Feldman LS, Vassiliou MC, Demyttenaere S, Hoover ML. Sim one, do one, teach one: considerations in designing training curricula for surgical simulation. J Surg Educ. 2011;68:421-7.

22. Schout BMA, Hendrikx AJM, Scheele F, Bemelmans BLH, Scherpbier AJJA. Validation and implementation of surgical simulators: a critical review of present, past, and future. Surg Endosc. 2010;24:536-46.

23. Xeroulis GJ, Park J, Moulton C-A, Reznick RK, Leblanc V, Dubrowski A. Teaching suturing and knot-tying skills to medical students: A randomized controlled study comparing computer-based video instruction and (concurrent and summary) expert feedback. Surgery 2007;141:442-9.

24. Palter VN, Grantcharov T, Harvey A, MacRae HM. Ex vivo technical skills training transfers to the operating room and enhances cognitive learning: a randomized controlled trial. Ann Surg. 2011;253:886-9. 